\title{
Cryo- Electron Tomography and Fluorescence Microscopy of Unicellular Algae in Vitreous Cryosections
}

\author{
Andrew Leis*, Lars Andrees*, Manuela Gruska*, Ashraf Al-Amoudi**, Anna Sartori*, Jacques \\ Dubochet** \& Wolfgang Baumeister* \\ * Max Planck Institute for Biochemistry, Department of Structural Biology, Am Klopferspitz 18a, \\ D-82152 Munich, Germany \\ ** University of Lausanne, Laboratory for Ultrastructural Analysis, Department of Biology, CH- \\ 1015 Lausanne, Switzerland
}

Cryo- electron tomography allows the visualisation of the internal structure of vitreous biological material at a resolution of 3-5 nm [1]. Physical fixation of cells and tissues by rapid freezing and maintenance of this state during tomography ensures optimal preservation of ultrastructure [2]. Due to the strong interaction of electrons with matter, vitreous samples thicker than ca. $0.8 \mu \mathrm{m}$ cannot be visualised in toto. We have combined mechanical sectioning ('cryosectioning') and subsequent tomographic analysis to visualise subcellular detail in the unicellular algae Cyanidioschyzon merolae and Cyanidium caldarium. The more general aim of our study is to determine the molecular architecture and spatial organisation of eukaryotic cells in a non-invasive manner.

C. merolae and C. caldarium have a primitive structure, with only one copy of each of the principle cellular organelles [3]. Tomograms of peripheral regions of intact $C$. merolae have shown the cell wall, adjacent chloroplast membranes, the edge of the cell nucleus, the Golgi apparatus, and associated macromolecular complexes (not shown). Tomograms of thick $(100-200 \mathrm{~nm})$ cryosections (Fig. 1) often revealed complete cross-sections of the nucleus, mitochondrion and chloroplast, as well as electron-dense, macromolecular complexes within the cytoplasm. Artefacts intrinsic to the mechanical sectioning process could be identified unambiguously, thus permitting confidence in the interpretation of e.g. membrane continuity within organelles.

The small size of $C$. merolae and C. caldarium ( $2 \mu \mathrm{m}$ and $4 \mu \mathrm{m}$, respectively) and robust nature conferred by a cell wall allows concentration of these organisms by mild centrifugation, leading to efficient recovery in cryosections. Clearly, many eukaryotic cells cannot tolerate this form of sample pre-treatment. If cell yields in cryosections are low, cryo- fluorescence microscopy can be used to determine the spatial distribution of cells within frozen sections (Fig. 2). The presence of autofluorescence following vitrification and cryosectioning demonstrates the possibility of detecting fluorescent antibody or quantum dot conjugates in cryosections. Using an extension to recently developed tomography software tools [4], areas of interest are recalled at the electron microscope, bypassing laborious manual searches and the associated risks of beam-induced specimen damage or contamination with crystalline ice. Although cryosectioning is notoriously problematic, the level of detail that could be observed in tomographic reconstructions demonstrates that it is instructive to perform cryo- electron tomography on cryosectioned, eukaryotic cells. Future investigations will determine the feasibility of applying pattern-recognition algorithms to cryosectioned cells.

\section{References}

[1] W. Baumeister, Biol. Chem. 385 (2004) 865.

[2] J. Dubochet and N. Sartori Blanc, Micron 32 (2001) 91.

[3] M. Matsuzaki et al., Nature 428 (2004) 653.

[4] S. Nickell et al., J. Struct. Biol. (2005) in press. 

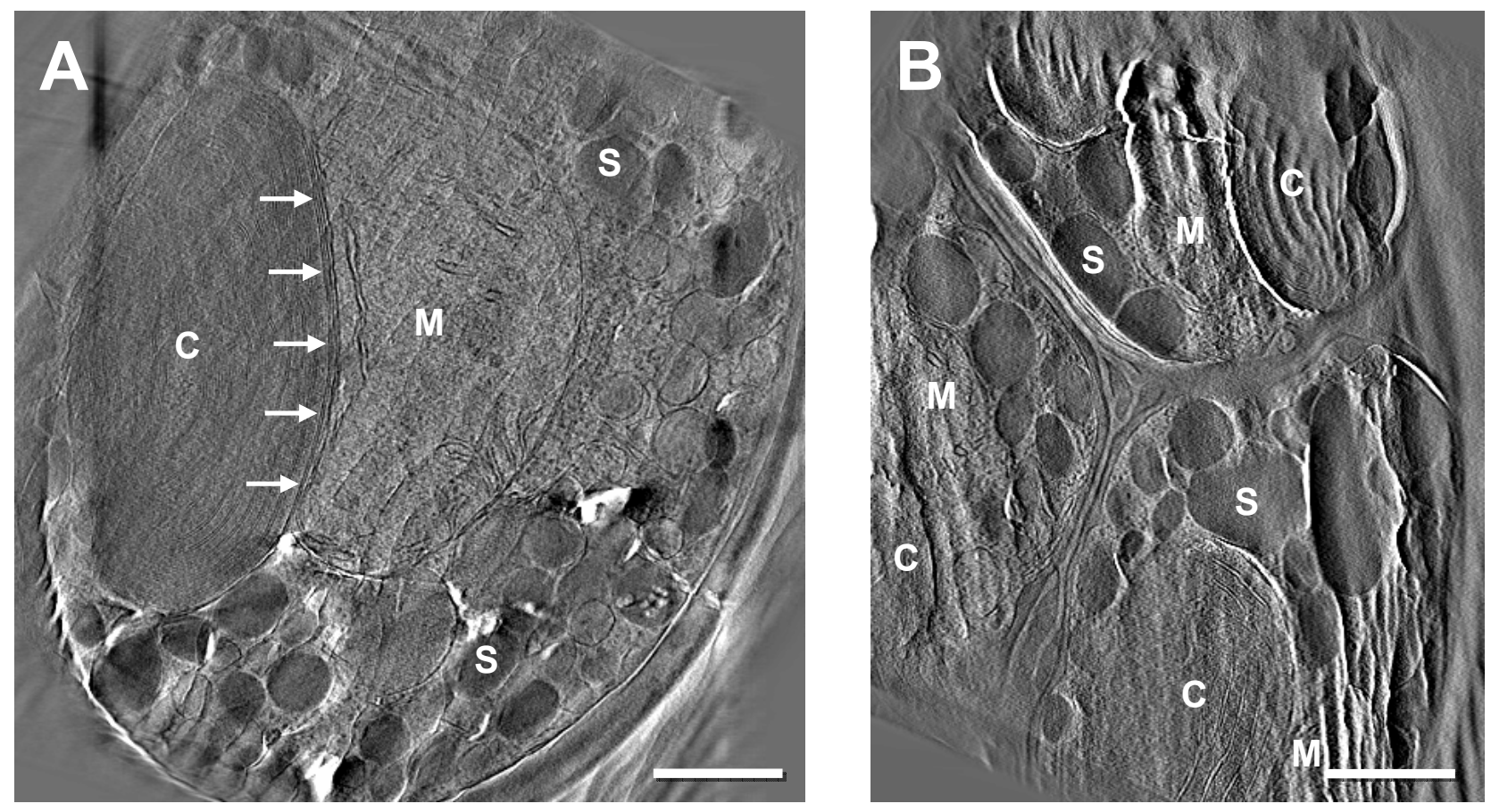

Figure 1. Reconstructed volumes from tomograms of cryosectioned algae. (A) Ten, superimposed projection images from a reconstructed tomogram of cryosectioned Cyanidioschyzon merolae. Note how the chloroplast and mitochondrion appear to be contiguous (arrows). The cells were concentrated by low-speed centrifugation $(4500 \times \mathrm{g})$ followed by two washes and resuspension in $20 \%$ dextran cryoprotectant. Labels indicate a chloroplast (C), mitochondrion (M), and starch granules (S). Scale bar $=500 \mathrm{~nm}$. (B) Projection image from a cryosectioned, dividing alga, Cyanidium caldarium. Unlike $C$. merolae, which divides by binary fission, $C$. caldarium divides into 4 daughter cells before segregation of the individual cells. Three of the four cells are visible in this cross-section. Labelled structures are as in (A). Scale bar $=500 \mathrm{~nm}$.

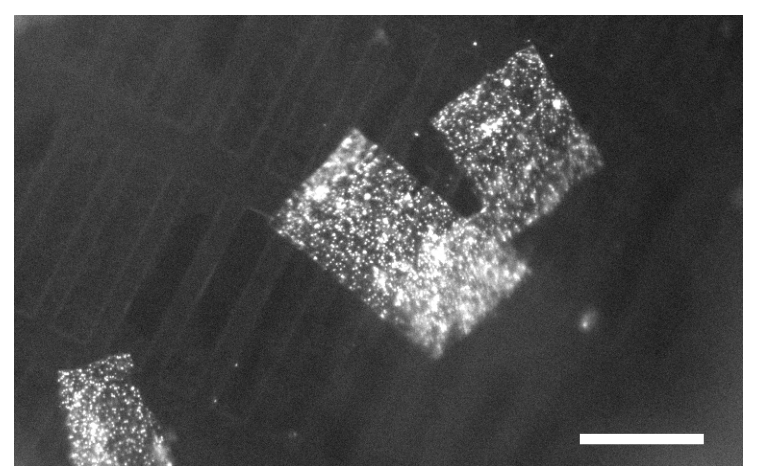

Figure 2. Cryo- fluorescence micrograph of $100 \mu \mathrm{m}$-thick cryosections containing autofluorescent algae. Each band comprises several individual sections. The cryosections were transferred to a carbon-coated $400 \times 100$ mesh TEM sample grid and mounted in a purpose-built apparatus with a liquid nitrogen reservoir. In the case of 'marker' grids, this approach allows the recording of coordinates and correlation with TEM images to recover objects of interest. Scale bar $=100 \mu \mathrm{m}$. 